\title{
BRST FORMULATION of 4-MONOPOLES
}

\author{
R. Gianvittorio, I.Martin and A. Restuccia \\ Departamento de Física, Universidad Simón Bolívar \\ Apartado postal 89000, Caracas 1080-A, Venezuela.
}

\begin{abstract}
A supersymmetric gauge invariant action is constructed over any 4dimensional Riemannian manifold describing Witten's theory of 4-monopoles. The topological supersymmetric algebra closes off-shell. The multiplets include the auxiliary fields and the Wess-Zumino fields in an unusual way, arising naturally from BRST gauge fixing. A new canonical approach over Riemann manifolds is followed, using a Morse function as an euclidean time and taking into account the BRST boundary conditions that come from the BFV formulation. This allows a construction of the effective action starting from gauge principles.
\end{abstract}

e-mail:ritagian@usb.ve,isbeliam@usb.ve,arestu@usb.ve 


\section{Introduction}

The non-perturbative analysis of Quantum Field Theory (QFT)is one of the main problems that has lately been discussed in different theories. In Superstring and Super Yang Mills theories by searching for a duality symmetry principle in perturbatively finite theories.In Quantum Gravity by using a loop description of spacetime. However, it is in Topological QFT (TQFT) that the non-perturbative analysis has provided the most striking results. In particular, the exact evaluation of correlation functions given rise to topological invariants of the base 4-manifolds, i.e the Donaldson invariants.

This non-perturbative analysis is sucessful because of the very particular structure of topological quantum effective actions. One may understand this stucture in terms of the symmetries underlying the topological field theories. To do so, one may start from a lagrangian independent of the metric on the base manifold with a huge group of symmetries, the "topological" symmetries. Then by BRST gauge fixing one arrives to the off-shell BRST invariant effective action describing the topological theory. At that level the analysis of the topological twisted supersymmetry becomes straightforward. Also, it is possible to see at the same time the role played by the "topological" symmetries in determining the linear dependence of the BRST charge on the conjugate momenta of the fields and ghosts. This is so in spite of the fact, that the theory is invariant under diffeomorphisms on the base 4-manifold. It is this feature which allows a complete non-perturbative analysis of the quantum theory.

The programme of studying topological invariants by starting from a TQFT was proposed by Witten in [1], who found an effective twisted supersymmetric topological action for the $S U(2)$ instantons and obtained the Donaldson invariants from the topological observables of the theory.In there, he also put forward the question as to what was the gauge action principle associated to his effective supersymmetric action. Several gauge principles have been proposed in the literature [2][3], which after BRST gauge fixing lead to Witten's effective action. The simplest one was given in [3]. The starting point is an action independent of the metric. This assures that the partition function will also be independent of metrics and provides all the supersymmetric structure from the BRST analysis. Moreover, it allows a more general construction than the one proposed in [1], including the supersymmetric auxiliary fields.

In this paper we construct in detail the gauge action principle for the topological theory of monopoles over 4-manifolds [4], first presented in [5]. We introduce a new canonical formulation over any Riemannian manifold which leads without any restrictive assumption on the manifold to a final covariant BRST effective action. In particular, it avoids the usual assumption that the base manifold is a product $R \times \Sigma_{3}$ in order to perform a canonical analysis. The final action we propose in section 4 , is invariant under an off-shell topological SUSY algebra. It includes the Wess Zumino fields as well as the auxiliary fields of the SUSY multiplet.The Wess Zumino fields have been considered in [6] using superfields constructed from a general analysis of the full twisted supersymmetry algebra. However, our construction is simpler and allows for an explicit presentation in terms of components.In [6] also, a topological quantum field theory was given using the Mathai-Quillen formalism. We compare both results at the end of sections 3 and 4 . 


\section{The gauge invariant action}

The $S U(2)$ topological quantum field theory of Witten [1] can be obtained from a twisted version of $\mathrm{N}=2$ supersymmetric Yang-Mills theory which arises directly by BRST gauge fixing of a Lagrangian involving only the curvature of the $S U(2)$ connection and an auxiliary 2-form [3]. As a consequence of the twisting there is no special requirement over the spin structure on the general differentiable 4-manifold and the quantum theory may be formulated starting from a general orientable riemannian 4-manifold. However, the construction of a gauge invariant action for Witten monopole equations requires from the beginning the existence of a $\operatorname{Spin}_{c}$ structure over the 4-manifold, luckily this existence is assured for any orientable riemannian manifold in four dimensions . In the case when the second Stiefel-Whitney class of the 4 -manifold is zero, i.e. $\omega_{2}=0$, the $S O(n)$ structure group of the tangent bundle can always be lifted to $\operatorname{Spin}(n)$ and, hence, it is possible to define the corresponding spin structure. In other cases when $\omega_{2}$ is reducible modulo two of an integral cohomology class $c_{1} \in H^{2}(X, Z)$, it is always possible to lift $S O(n)$ to $\operatorname{Spin}_{c}(n)=\operatorname{Spin}(n) \times_{Z_{2}} U(1)$ and to define a $\operatorname{Spin}_{c}$ structure. As said before, over any orientable 4-manifold a $\operatorname{Spin}_{c}$ structure can always be constructed as $\omega_{2}$ is always reducible modulo two of the integer Chern class [7]. This property is not valid in general for manifolds of dimension $d>4$ but holds perfectly for orientable 4-manifolds. It is this unique property which allows the Witten construction over a general riemannian 4-manifold.

The action over a general differentiable 4-manifold $\mathrm{X}$ we propose is given by

$$
S=\frac{1}{8} \int_{X}\left(F_{\mu \nu}+B_{\mu \nu}+\frac{i}{2} \bar{M} \Gamma_{\mu \nu} M\right)\left(F_{\rho \sigma}+B_{\rho \sigma}+\frac{i}{2} \bar{M} \Gamma_{\rho \sigma} M\right) d x^{\mu} \wedge d x^{\nu} \wedge d x^{\rho} \wedge d x^{\sigma},
$$

The field $F_{\mu \nu}$ is the curvature associated to the $U(1)$ connection $A_{\mu}$ over a complex line bundle $L, B_{\mu \nu} d x^{\mu} \wedge d x^{\nu}$ is an independent auxiliarly 2-form. $M$ and its complex conjugate $\bar{M}$ are sections of $S^{+} \otimes L$ and $S^{-} \otimes L^{-1}$ respectively, where $L^{-1}$ is the complex conjugate bundle of $L$ and $S^{+}$is one of the irreducible parts of the spinor bundle $S$. For any even manifold with a $\operatorname{Spin}_{c}$ structure there is always a unique spinor bundle $S$ associated to a representation of $\operatorname{Spin}_{c}$ that splits into a direct sum $S(X)=S^{+}(X) \oplus S^{-}(X)$. The Clifford matrices $\Gamma_{\mu}$ satisfy $\left\{\Gamma_{\mu}, \Gamma_{\nu}\right\}=2 g_{\mu \nu}$. The local symmetries of this action are given by the following infinitesimal transformations:

$$
\begin{gathered}
\delta_{\lambda} A_{\mu}=\mathcal{D}_{\mu} \Lambda, \quad \delta_{\lambda} B_{\mu \nu}=0, \quad \delta_{\lambda} M=i \Lambda M ; \\
\delta_{\epsilon} A_{\mu}=\epsilon_{\mu}, \quad \delta_{\epsilon} M=0, \quad \delta_{\epsilon} B_{\mu \nu}=-\mathcal{D}_{[\mu} \epsilon_{\nu]} ; \\
\delta_{\theta} M^{A}=\theta^{A}, \quad \delta_{\theta} B_{\mu \nu}=\left(-\frac{i}{2} \bar{\theta} \Gamma_{\mu \nu} M-\frac{i}{2} \bar{M} \Gamma_{\mu \nu} \theta\right) ;
\end{gathered}
$$

where $\Lambda$ is the local infinitesimal parameter associated to the gauge group $U(1), \epsilon_{\mu}$ and $\theta^{A}$ are the local infinitesimal parameters associated to differentiable deformations in the space of $U(1)$ connections and of sections of $S^{+} \otimes L$ respectively. We require $\epsilon_{\mu}$ to be globally restricted by the condition that $A_{\mu}+\delta_{\epsilon} A_{\mu}$ must also be a connection 
on the $U(1)$ principle bundle. $\epsilon_{\mu}$ may eliminate any local excitation mode of $A_{\mu}$, but because of the above global restriction it can not change the cohomology class of $F_{\mu \nu}$. To show this point, we notice that we may always find a real parameter $\lambda$ such that $A_{\mu}+\epsilon_{\mu}$ may be written in the following way

$$
A_{\mu}+\epsilon_{\mu}=(1-\lambda) A_{\mu}+\lambda C_{\mu}
$$

where $C_{\mu} d x^{\mu}$ is a particular 1 -form connection in $\mathcal{A}$, the convex space of connections. We thus have

$$
\epsilon_{\mu}=\lambda\left(C_{\mu}-A_{\mu}\right)
$$

this means that $\epsilon_{\mu}$ is invariant under transitions on the intersection of two neighborhoods on the principal bundle. Also, under the transformation

$$
A_{\mu} \rightarrow A_{\mu}+\epsilon_{\mu}
$$

the curvature 2 -form changes by

$$
F \rightarrow F+d \epsilon
$$

but because of the invariance property of $\epsilon_{\mu}$ under transitions on the principal bundle $d \epsilon$ is an exact 2 -form, i.e. $d \epsilon$ may change $F$ within a cohomology class only. It can not annihilate, for example, a Dirac monopole curvature 2-form. (2.2), (2.3) and (2.4) together with the global restriction on $\epsilon_{\mu}$ define the gauge symmetries of our theory. That is, in the functional integral we integrate over the gauge inequivalent classes of the fields, where two elements of the same class are related by a finite transformation generated by the group of gauge symmetries.

We may construct then the effective action of the gauge invariant action (2.1) by imposing a gauge fixing condition on the 1 -form $A$. However, it is difficult to fix $A$ taking into account a global restriction on $\epsilon_{\mu}$ and at the same time obtaining a covariant effective action under (2.2). Nevertheless, it is possible to satisfy both conditions by imposing a gauge fixing condition on the antisymmetric field $B_{\mu \nu}$. At first sight it would seem that we could eliminate completely the 2-form $F$ field in the functional integral by performing a change of integration variable on the 2 -form $B$,

$$
\widetilde{B}=B+F
$$

ending up with a trivial theory. However, this is not the case since in the functional integral we must integrate on the gauge equivalent classes of antisymmetric fields. We return to this point in eq. (3.12).

To construct the effective action corresponding to the gauge fixing associated to transformations (2.3), we proceed as follows. First, we notice that (2.3) contains the $U(1)$ gauge transformations (2.2). Since we would like to preserve the invariance under (2.2), we can not fix $\epsilon_{\mu}$ completely, i.e. we must leave its longitudinal component undetermined. Second, we need a covariant condition which may deformed to zero by using the three remaining degrees of freedom in $\epsilon_{\mu}$. The appropiate geometrical object is

$$
B_{\mu \nu}^{+} \equiv \frac{1}{2}\left(B_{\mu \nu}+\frac{1}{2} g^{1 / 2} \epsilon_{\mu \nu \sigma \rho} B^{\sigma \rho}\right)
$$


We have from (2.3)

$$
\delta_{\epsilon} B_{0 i}^{+}=-\frac{1}{2}\left(\mathcal{D}_{0} \epsilon_{i}-D_{i} \epsilon_{0}+\frac{1}{2} g^{1 / 2} \epsilon_{0 i j k} \mathcal{D}^{j} \epsilon^{k}\right)
$$

given $\epsilon_{0}$ and $\delta B_{0 i}^{+},(2.5)$ is a first order differential equation in $\epsilon_{i}$ which has always a solution, allowing local infinitesimal deformations of $B_{0 i}^{+}$. Since $B_{\mu \nu}$ and $\epsilon_{\mu \nu}$ do not change under a transition from one chart to another on the principal bundle, the deformations may be extended globally. Next, since $B_{0 i}^{+}=0$ implies $B_{i j}^{+}=0$, we may impose the covariant gauge fixing condition

$$
B_{\mu \nu}^{+}=0
$$

We notice that by restricting $\epsilon_{i}$, we do not constrain the longitudinal part of $\epsilon_{\mu}$. In fact, $\epsilon_{\mu}$ may be decomposed into

$$
\epsilon_{\mu}=\epsilon_{\mu}^{T}+\mathcal{D}_{\mu} \epsilon^{L}
$$

where $\mathcal{D}^{\mu} \epsilon_{\mu}^{T}=0$. The longitudinal part of $\epsilon_{\mu}$ is determined by taking

$$
\mathcal{D}^{\mu} \epsilon_{\mu}=\mathcal{D}^{\mu} \mathcal{D}_{\mu} \epsilon^{L}
$$

Given $\epsilon_{i}, \mathcal{D}^{\mu} \epsilon_{\mu}$ is still completely undetermined because of the presence of $\epsilon_{0}$ which was not restricted by (2.6). $\epsilon^{L}$ is the gauge parameter associated to transformations (2.2), the ordinary $U(1)$ gauge transformations on the principal bundle. We may now consider the gauge fixing related to transformations (2.4). Taking into account the global harmonic modes over $\mathrm{X}$, we may consider

$$
\Gamma^{\mu} \mathcal{D}_{\mu} M=0
$$

where $\Gamma^{\mu} \mathcal{D}_{\mu}$ is the Dirac operator that maps sections of $S^{+} \otimes L$ to sections of $S^{-} \otimes L$. The field equations associated to (2.1) are

$$
F_{\mu \nu}+B_{\mu \nu}+\frac{i}{2} \bar{M} \Gamma_{\mu \nu} M=0
$$

by using (2.6), the field equations then reduce to

$$
\begin{aligned}
& F_{\mu \nu}^{-}+B_{\mu \nu}^{-}=0, \\
& F_{\mu \nu}^{+}+\frac{i}{2} \bar{M} \Gamma_{\mu \nu} M=0 .
\end{aligned}
$$

From (2.6) and (2.8a) we determine the auxiliarly field $B_{\mu \nu}$. The eqs. (2.8b) and (2.7) are the monopole equations obtained in [4]. 


\section{BRST gauge fixing}

We now construct the BRST invariant action with the following procedure generalizing the standard one [8]. We start with a canonical formulation of (2.1) obtaining a BRST invariant effective action, after a covariant gauge fixing and integration of the conjugate momenta in the functional integral. This action is manifestly covariant under general coordinate transformations. The base manifold X we are considering is a compact Riemannian one. Consequently, it does not satisfy the usually assumed requirement of being globally a product $R \times \Sigma_{3}$ as is the case in an ordinary canonical formulation. We need to proceed differently here. We choose two points on $\mathrm{X}$, namely $A$ and $B$. It is well known that a polar Morse function always exist globally on any 4-manifold $\mathrm{X}$, with minimum and maximum height at $A$ and $B$ respectively. The Morse function is defined by embedding $\mathrm{X}$ on $R_{N}$ and considering an appropiate direction on $R_{N}$. The projection on this direction defines a height $\tau$ as the Euclidean "time" over X.

We construct now the canonical formulation using $\tau$ as one of the local coordinates over X. We may decompose locally $\mathrm{X}$ as a product $R \times \Sigma_{3}$ between consecutive $\tau_{c}$ 3manifolds, where $\Sigma_{3}$ is a compact three manifold and $\tau_{c} 3$-manifolds define limiting manifolds that separate compact 3-manifolds with different number of connected components. In this way, it is enough to decompose $\mathrm{X}$ into the "cilinders" determined by the "evolution" of the disconnected parts of $\tau=$ constant submanifold $\Sigma_{3}$ between two consecutive $\tau_{c}$ manifolds, as it is indicated in figure 1 , where $\tau_{1}$ and $\tau_{3}$ are $\tau_{c}$ 3-manifolds.

Figure 1

In the construction of a BRST invariant effective action, there are boundary conditions that must be imposed on the BRST charge $\Omega$ at $\tau=\tau_{i}$ and $\tau=\tau_{f}$ to have a functional integral independent of the gauge fixing condition [8]. In our formulation those points correspond to $A$ and $B$ respectively. The boundary conditions are

$$
\left.\left[\Omega-<\pi \frac{\delta \Omega}{\delta \pi}>-<\eta_{A} \frac{\delta \Omega}{\delta \eta_{A}}>-<\bar{\eta}_{A} \frac{\delta \Omega}{\delta \bar{\eta}_{A}}>-<\mu^{1 i} \frac{\delta \Omega}{\delta \mu^{1 i}}>-<\mu^{1} \frac{\delta \Omega}{\delta \mu^{1}}>\right]\right|_{\tau_{i}} ^{\tau_{f}}=0
$$


In here $\langle\ldots$.$\rangle means integration on \Sigma_{3}$. These boundary conditions are imposed usually on the ghost fields sector associated to the constraints that are not linear and homogeneous in the momenta. This is the typical situation that occurs in the Hamiltonian constraint in a diffeomorphic invariant theory. Since (2.1) is invariant under diffeomorphisms over $\mathrm{X}$, we must pay special attention to these boundary conditions in our formulation. The point is that we may choose $A$ and $B$ arbitrarily over $\mathrm{X}$, they are not natural limits as in a Minkowskian spacetime, consequently, any condition on the ghost fields at these points may ruin the possibility of a covariant construction. Fortunately, as we will show those boundary conditions are satisfied identically in the topological theory we propose, for any pair of points chosen. And so, we are able to end up with a correct canonical as well as a manifestly covariant formulation under general coordinate and off-shell supersymmetric transformations. We discuss the canonical formulation and the boundary conditions for other topological theories elsewhere. The canonical form of the action is

$$
\begin{gathered}
S=\sum_{L} S_{L}, \\
S_{L}=\int_{U_{L}} d^{4} x\left[\dot{A}_{i} \epsilon^{i j k}\left(F_{j k}+B_{j k}+\frac{i}{2} \bar{M} \Gamma_{j k} M\right)+A_{0} \mathcal{D}_{i}\left(\epsilon^{i j k}\left(F_{j k}+B_{j k}+\frac{i}{2} \bar{M} \Gamma_{j k} M\right)\right)+\right. \\
\left.\left(B_{0 i}+\frac{i}{2} \bar{M} \Gamma_{0 i} M\right) \epsilon^{i j k}\left(F_{j k}+B_{j k}+\frac{i}{2} \bar{M} \Gamma_{j k} M\right)\right],
\end{gathered}
$$

The eq. (3.2) yields the canonical conjugate momenta to $A_{i}, \pi^{i}$ which is a density under diffeomorphisms,

$$
\pi^{i}=\epsilon^{i j k}\left(F_{j k}+B_{j k}+\frac{i}{2} \bar{M} \Gamma_{i j} M\right)
$$

$A_{0}$ and $B_{0 i}+\frac{i}{2} \bar{M} \Gamma_{0 i} M$ are the Lagrange multipliers associated respectively to the constraints

$$
\begin{aligned}
& \phi \equiv \mathcal{D}_{i} \pi^{i}=0, \\
& \phi^{i} \equiv \pi^{i}=0
\end{aligned}
$$

the other constraints are given by

$$
\begin{aligned}
& \phi^{A} \equiv \eta_{A}=0, \\
& \bar{\phi}^{A} \equiv \bar{\eta}_{A}=0,
\end{aligned}
$$

where $\bar{\eta}_{A}$ and $\eta_{A}$ are the conjugate momenta to $M^{A}$ and $\bar{M}^{A}$ respectively.

All the constraints conmute, however (3.3a) and (3.3b) are not independent. The reducibility matrix is given by

$$
a \equiv\left(\mathcal{D}_{i},-1\right) .
$$


To construct the BRST charge we follow [8] and introduce the minimal sector of the extended phase space expanded by the conjugate pairs:

$$
\left(A_{i}, \pi^{i}\right) ;\left(M^{A}, \bar{\eta}_{A}\right) ;\left(\bar{M}^{A}, \eta_{A}\right) ;\left(C_{1}, \mu^{1}\right),\left(C_{1 i}, \mu^{1 i}\right) ;\left(C_{11}, \mu^{11}\right) ;\left(C^{A}, \bar{\mu}_{A}\right)
$$

where we have introduced the ghost and antighost associated to the first class constraints.

The off-shell nilpotent BRST charge is then given by:

$$
\begin{gathered}
\Omega=\sum_{a} \Omega_{a} \\
\Omega_{a}=\int_{\Sigma_{3}^{(a)}} d^{3} x\left(-\left(\mathcal{D}_{i} C_{1}\right) \pi^{i}+C_{1 i} \pi^{i}+2 i C^{A} \bar{\eta}_{A}-2 i \bar{C}^{A} \eta_{A}-\left(\mathcal{D}_{i} C_{11}\right) \mu^{1 i}-C_{11} \mu^{1}\right),
\end{gathered}
$$

$\Omega_{a}$ is the BRST charge for every $a$ connected component of $\Sigma_{3}$. It is straightforward to check from (3.6) that the boundary conditions (3.1) are satisfied identically. Hence the necessary condition to get a covariant formalism mentioned at the beginning of this section is fulfilled.

We now define the non minimal sector of the extended phase space [8]. It contains extra ghosts, antighosts and Lagrange multipliers. First we introduce the C-fields

$$
C_{m}, C_{m i} ; \quad C_{m n}, C_{m n i} ; \quad m, n=1,2,3
$$

where at least one of the indices $m, n$ take the values 2 or 3 . In addition to these ghost, antighost and Lagrange multiplier fields we introduce the $\lambda$ and $\theta$ fields (Lagrange multipliers), also in the non minimal sector,

$$
\begin{aligned}
& \lambda_{1}^{0}, \quad \lambda_{1 i}^{0} ; \quad \lambda_{1 m}^{0} ; \quad m=1,2,3 \\
& \lambda_{11}^{1} ; \\
& \theta_{1}^{0}, \theta_{1 i}^{0} ; \quad \theta_{1 m}^{0} ; \quad m=1,2,3 \\
& \theta_{11}^{1} .
\end{aligned}
$$

In this notation the 1 subscripts denote ghost associated to a gauge symmetry of the action, the 2 subscripts denote antighost associated to a gauge fixing condition in the effective action and the 3 subscripts denote Lagrange multipliers associated to a gauge fixing condition. The canonical BRST invariant effective action is then given by:

$$
\begin{aligned}
S_{e f f}=\sum_{L} \int_{U_{L}} d^{4} x & {\left[\pi^{i} \dot{A}_{i}+\bar{\eta}_{A} \dot{M}^{A}+\eta_{A} \dot{\bar{M}}^{A}+\mu^{1} \dot{C}_{1}+\mu^{1 i} \dot{C}_{1 i}+\right.} \\
& \mu^{11} \dot{C}_{11}+\bar{\mu}_{A} \dot{C}^{A}+\mu_{A} \dot{\bar{C}}^{A}+ \\
& \left.\widehat{\delta}\left(\lambda_{1}^{0} \mu^{1}+\lambda_{1 i}^{0} \mu^{1 i}+\lambda_{11}^{1} \mu^{11}+\lambda^{A} \bar{\mu}_{A}+\bar{\lambda}^{A} \mu_{A}\right)+L_{G F+F P}\right],
\end{aligned}
$$


where

$$
L_{G F+F P}=\widehat{\delta}\left(C_{2} \chi_{2}+C_{2 \mu \nu} \chi_{2}^{\mu \nu}+C_{2}^{\dot{A}} \bar{\chi}_{\dot{A}}+\bar{C}_{2}^{\dot{A}} \chi_{\dot{A}}\right)+\widehat{\delta}\left(C_{12} \chi_{12}\right)+\widehat{\delta}\left(\lambda_{12}^{0} \Lambda_{2}+\theta_{12}^{0} \Theta_{2}\right)
$$

is the sum of the generalizations of the Fadeev-Popov and gauge fixing terms. In (3.8) $\chi_{2}, \chi_{2}^{\mu \nu}, \chi_{\dot{A}}$ and $\bar{\chi}_{\dot{A}}$ are the gauge fixing functions associated to the constraints (3.3), while $\chi_{12}, \Lambda_{2}$ and $\Theta_{2}$ are gauge fixing functions associated to the reducibility problem. They must fix the longitudinal part of the fields $C_{1 \mu}, \lambda_{1}^{0}$ and $\theta_{1}^{0}$. Notice that $C_{2 \mu \nu}$ is a self-dual density. Also, all antighosts in (3.8) are densities. The BRST transformation for the canonical variables is given by

$$
\widehat{\delta} Z=(-1)^{\epsilon_{z}}\{Z, \Omega\}
$$

where $\epsilon_{z}$ is the grassmanian parity of $Z$. The BRST transformation of the variables of the non minimal sector are given in [8]. After integration of the auxiliarly sector we finally choose gauge fixing functions that may be written in a covariant form as

$$
\begin{aligned}
& \chi_{2}=\mathcal{D}_{\mu} A^{\mu}-\frac{\alpha}{2} g^{-\frac{1}{2}} C_{3}, \\
& \chi_{2}^{\mu \nu}=\frac{1}{4}\left(\frac{1}{2} g^{-\frac{1}{2}} \epsilon^{\mu \nu \sigma \rho} B_{\sigma \rho}+B^{\mu \nu}\right), \\
& \chi_{12}=\mathcal{D}^{\mu} C_{1 \mu}+\frac{1}{2}\left(-\bar{C}^{A} M_{A}+\bar{M}^{A} C_{A}\right), \\
& \bar{\chi}_{\dot{A}}=-\frac{i}{2} \mathcal{D}_{A \dot{A}} \bar{M}^{A}+\rho g^{-\frac{1}{2}} \bar{C}_{3 \dot{A}}, \\
& \chi_{\dot{A}}=-\frac{i}{2} \mathcal{D}_{A \dot{A}} M^{A}+\rho g^{-\frac{1}{2}} C_{3 \dot{A}},
\end{aligned}
$$

where $C_{1 \mu}=\left(-\lambda_{11}^{0}, C_{1 i}\right)$ and $\rho$ is an arbitrary real parameter. After elimination of all conjugate momenta in the functional integral, the BRST transformation rules of all the 
remaining geometrical objects are covariant and take the form

$$
\begin{aligned}
& \widehat{\delta} A_{\mu}=-\mathcal{D}_{\mu} C_{1}+C_{1 \mu}, \\
& \widehat{\delta} C_{1}=C_{11}, \\
& \widehat{\delta} C_{1 \mu}=\mathcal{D}_{\mu} C_{11}, \\
& \widehat{\delta} C_{11}=0, \\
& \widehat{\delta} C_{2}=C_{3}, \\
& \widehat{\delta} C_{3}=0, \\
& \widehat{\delta} C_{2 \mu \nu}=C_{3 \mu \nu}, \\
& \widehat{\delta} C_{3 \mu \nu}=0, \\
& \widehat{\delta} C_{12}=C_{13}, \\
& \widehat{\delta} C_{13}=0, \\
& \widehat{\delta} C^{A}=0, \\
& \widehat{\delta} M^{A}=-2 i C^{A}, \\
& \widehat{\delta} C_{2}^{\dot{A}}=C_{3}^{\dot{A}}, \\
& \widehat{\delta} C_{3}^{\dot{A}}=0, \\
& \widehat{\delta} B_{\mu \nu}^{+}=-\widehat{\delta} F_{\mu \nu}^{+}-\frac{i}{2} \widehat{\delta}\left(\bar{M} \Gamma_{\mu \nu} M\right)
\end{aligned}
$$

$C_{2 \mu \nu}$ and $C_{3 \mu \nu}$ are self dual fields.

The BRST invariant action under the off shell nilpotent algebra (3.11) follows then from (3.7), it is given by

$$
\begin{gathered}
\widetilde{S}=\frac{1}{8} \int_{X}\left(F_{\mu \nu}+B_{\mu \nu}+\frac{i}{2} \bar{M} \Gamma_{\mu \nu} M\right)\left(F_{\rho \sigma}+B_{\rho \sigma}+\frac{i}{2} \bar{M} \Gamma_{\rho \sigma} M\right) d x^{\mu} \wedge d x^{\nu} \wedge d x^{\rho} \wedge d x^{\sigma} \\
+\int_{X} d^{4} x\left[\widehat{\delta}\left(C_{2} \chi_{2}+C_{2 \mu \nu} \chi_{2}^{\mu \nu}+C_{2}^{\dot{A}} \chi_{\dot{A}}+\bar{C}_{2}^{\dot{A}} \bar{\chi}_{\dot{A}}\right)+\widehat{\delta}\left(C_{12} \chi_{12}\right)\right],
\end{gathered}
$$

In the associated functional integral we may change variables by considering

$$
\widehat{B_{\mu \nu}}=F_{\mu \nu}+B_{\mu \nu}+\frac{i}{2} \bar{M} \Gamma_{\mu \nu} M
$$

ending up with the following effective action

$\widetilde{S_{1}}=\frac{1}{8} \int_{X} \widehat{B_{\mu \nu}} \widehat{B_{\rho \sigma}} d x^{\mu} \wedge d x^{\nu} \wedge d x^{\rho} \wedge d x^{\sigma}+\int_{X} d^{4} x\left[\widehat{\delta}\left(C_{2} \chi_{2}+C_{2 \mu \nu} \chi_{2}^{\mu \nu}+C_{2}^{\dot{A}} \chi_{\dot{A}}+\bar{C}_{2}^{\dot{A}} \bar{\chi}_{\dot{A}}\right)+\widehat{\delta}\left(C_{12} \chi_{12}\right)\right]$,

where we have to rewrite $\chi_{2 \mu \nu}$ as a function of $\widehat{B_{\mu \nu}}$,

$$
\chi_{2 \mu \nu}=\frac{1}{2}\left(\widehat{B_{\mu \nu}^{+}}-F_{\mu \nu}^{+}-\frac{i}{2} \bar{M} \Gamma_{\mu \nu} M\right)
$$


We are able now to show the topological structure of the partition function $I$ associated to $\widetilde{S}$. In fact the only dependence on the background metric is through gauge fixing conditions. Using the BFV theorem [8], the functional derivative of $I$ with respect to $\chi$ yields zero,

$$
\frac{\delta I}{\delta \chi}=0
$$

where $\chi$ is any of the set of gauge fixing conditions (3.10), hence

$$
\frac{\delta I}{\delta g_{\mu \nu}}=\frac{\delta I}{\delta \chi} \frac{\delta \chi}{\delta g_{\mu \nu}}=0
$$

showing that the theory defined by (2.1) is a topological one.

Eliminating $\widehat{B_{\mu \nu}}$ from the effective action, we obtain after functional integration

$$
\begin{aligned}
\widetilde{S_{2}}=\int_{X} d^{4} x g^{\frac{1}{2}}[ & -\frac{1}{4}\left(g^{-\frac{1}{2}} C_{3 \mu \nu}+F_{\mu \nu}^{+}+\frac{i}{2} \bar{M} \Gamma_{\mu \nu} M\right)^{2}+\frac{1}{4}\left(F_{\mu \nu}^{+}+\frac{i}{2} \bar{M} \Gamma_{\mu \nu} M\right)^{2}-g^{-\frac{1}{2}} C_{2 \mu \nu} \widehat{\delta} \chi_{2}^{\mu \nu} \\
& \left.+g^{-\frac{1}{2}} \widehat{\delta}\left(C_{2} \chi_{2}+C_{2}^{\dot{A}} \chi_{\dot{A}}+\bar{C}_{2}^{\dot{A}} \bar{\chi}_{\dot{A}}+C_{12} \chi_{12}\right)\right]
\end{aligned}
$$

$\widetilde{S_{2}}$ is invariant under the off-shell nilpotent BRST transformation (3.11). If we eliminate $C_{3 \mu \nu}$ by functional integration we obtain the action $S$, invariant under the algebra (3.11) with the substitution

$$
g^{-\frac{1}{2}} C_{3 \mu \nu}=-F_{\mu \nu}^{+}-\frac{i}{2} \bar{M} \Gamma_{\mu \nu} M,
$$

the algebra closes now only on-shell. After some calculations involving the $\rho$ dependent terms we get the action

$$
S=S_{0}+S_{1}+S_{2}+S_{3}
$$

where

$$
\begin{aligned}
& S_{0}=< \frac{1}{2} F^{+A B} F_{A B}^{+}+g^{\mu \nu} \mathcal{D}_{\mu} \bar{M}^{A} \mathcal{D}_{\nu} M_{A}+\frac{1}{4} R \bar{M}^{A} M_{A}-\frac{1}{8} \bar{M}^{(A} M^{B)} \bar{M}_{(A} M_{B)}> \\
& S_{1}=<C_{2}^{\mu \nu} \mathcal{D}_{\mu} C_{1 \nu}+C_{13} \mathcal{D}_{\mu} C_{1}^{\mu}+C_{12} \mathcal{D}_{\mu} \mathcal{D}^{\mu} C_{11}> \\
& S_{2}=<-C_{2}^{A B}\left(\bar{M}_{(A} C_{B)}+\bar{C}_{(A} M_{B)}\right)-\bar{C}_{2}^{\dot{A}} \mathcal{D}_{A \dot{A}} C^{A}-\bar{C}^{A} \mathcal{D}_{A \dot{A}} C_{2}^{\dot{A}} \\
&+\frac{1}{2}\left(\bar{M}^{A} C_{1 A \dot{A}} C_{2}^{\dot{A}}+\bar{C}_{2}^{\dot{A}} C_{1 A \dot{A}} M^{A}\right)-\frac{1}{2} C_{13}\left(\bar{C}^{A} M_{A}-\bar{M}^{A} C_{A}\right) \\
&+2 i C_{12} \bar{C}^{A} C_{A}-\frac{1}{2} \bar{M}^{A} \sigma_{A \dot{A}}^{\mu}\left(D_{\mu} C_{1}\right) C_{2}^{\dot{A}}-\frac{1}{2} \bar{C}_{2}^{\dot{A}} \sigma_{A \dot{A}}^{\mu}\left(D_{\mu} C_{1}\right) M^{A}>
\end{aligned}
$$

and finally

$$
S_{3}=<C_{3}\left(\mathcal{D}_{\mu} A^{\mu}-\frac{\alpha}{2} g^{-\frac{1}{2}} C_{3}\right)+C_{2} \mathcal{D}_{\mu} \mathcal{D}^{\mu} C_{1}-C_{2} \mathcal{D}_{\mu} C_{1}^{\mu}>
$$


where $<\ldots .>$ denotes integration on the 4-manifold $\mathrm{X}$ with the measure $g^{\frac{1}{2}}$ in (3.13), and $\rho$ has been taken as $\frac{1}{8}$ in order to cancel terms of the form $F^{+} \bar{M} M$. In these expressions we have rewritten the objects with world indices in terms of the corresponding ones with spinorial indices.

$S_{0}$ corresponds to the action used by Witten in deriving the vanishing theorems in [4]. While $S_{1}+S_{2}+S_{3}$ are the contributions of the ghost and antighost fields in order to have a BRST invariant action. The action $S_{0}$ agrees with the ghostless sector of the gauge fixed action proposed in [6]. In there also, the action is not invariant under the SUSY transformation rules given, unless one takes

$$
g^{-\frac{1}{2}} \widehat{\delta} C_{2 \mu \nu}=-\left(F_{\mu \nu}^{+}+\frac{i}{2} \bar{M} \Gamma_{\mu \nu} M\right)
$$

which arises from the general procedure we consider. Otherwise we agree with [6]. In (3.15) there are terms involving $C_{1}$ which are not present in the action given in [6] because the latter is invariant under BRST transformations which close only modulo gauge transformations. While the action we present in (3.12) is invariant under an off-shell nilpotent charge.

In order to compare with the formulation in [1], one may perform the change of variables:

$$
\begin{aligned}
& \psi_{\mu}=-i C_{1 \mu}, \\
& \phi=i C_{11}, \\
& \eta=-C_{13}, \\
& \lambda=-2 i C_{12}, \\
& \chi_{\mu \nu}=-C_{2 \mu \nu}, \\
& \mu^{A}=C^{A}, \\
& v^{\dot{A}}=2 i C_{2}^{\dot{A}} .
\end{aligned}
$$

showing that, after the reduction from $S U(2)$ to $U(1)$, the sectors associated to the gauge SUSY multiplet agree. The last two changes of variables allow direct comparison with [6].

\section{Topological supersymmetry}

The action (3.12) is invariant under the off-shell BRST transformations (3.11) and under general coordinate transformations over $M_{4}$ It is straightforward to obtain also a gauge invariant action. In fact the action

$$
\widehat{S}=S_{0}+S_{1}+S_{2}
$$

is still invariant under the above transformations and additionally under the gauge transformations on a $U(1)$ principle bundle. This is so, because $S_{3}$ arises from the 
gauge fixing plus Fadeev-Popov associated to the gauge invariance of the original action (2.1).More explicitly,

$$
S_{3}=<\widehat{\delta}\left(C_{2} \chi_{2}\right)>
$$

and hence it is BRST invariant on its own as well as under general coordinate transformations.

In [6], the full twisted supersymmetric algebra was constructed using a redefinition of the $\mathrm{SO}_{4}$ generators in which an identification of the isospin indices as right handed spin indices was performed

$$
\widetilde{J_{A B}}=J_{A B}-2 i T_{A B}
$$

It was shown there that there is a unique (up to a global factor) linear combination of the SUSY generators $Q_{a \alpha}$ such that it behaves as a scalar, under the new $\mathrm{SO}_{4}$ generators (4.3), satisfying $Q^{2}=0$. (3.11) is a realization of this nilpotent supersymmetric generator with an unusual Wess-Zumino auxiliary field structure with respect to the standard SUSY one [9].

In [6], an off-shell superfield realization of twisted supersymmetry resembling the Euclidean SUSY realization was introduced in terms of odd grassmannian coordinates $\theta$ and $\theta^{\alpha \beta}$. However, to obtain the component action a Wess-Zumino gauge is introduced.The SUSY algebra may then close only modulo field dependent gauge transformations. The Wess-Zumino auxiliary fields introduced in the superfields $W$ and $V$ are the twisted version of the usual ones [9]. What we show in (3.11) is that the off-shell closure of the subalgebra generated by the nilpotent charge $Q$ may be obtained by introducing only the $C_{1}$ auxiliary field. Moreover (3.12) is the invariant SUSY action associated to that realization.

We show now how to obtain the twisted SUSY algebra, in a Wess-Zumino gauge, from our nilpotent BRST algebra. Let us consider the transformation law for $A_{\mu}$. We define the SUSY transformation by

$$
\delta A_{\mu}:=\widehat{\delta} A_{\mu} \mid C_{1}=0
$$

we then have from $(3.10)$

$$
\begin{aligned}
\delta A_{\mu} & =C_{1 \mu} \\
\delta \delta A_{\mu} & =\delta C_{1 \mu}=\mathcal{D}_{\mu} C_{11} .
\end{aligned}
$$

The SUSY algebra thus closes up to a gauge transformation generated by $C_{11}$ as required.

The SUSY transformation for $M^{A}$ may be obtained by considering an equivalent BRST formulation to (3.5). Instead of considering the constraint (3.2a) we may take equivalently

$$
\mathcal{D}_{i} \pi^{i}+M^{A} \bar{\eta}_{A}+\bar{M}^{A} \eta_{A}=0
$$


The associated BRST charge is then given by

$$
\begin{aligned}
\Omega_{a}=\int_{\Sigma_{3}^{(a)}} d^{3} x( & -\left(\mathcal{D}_{i} C_{1}\right) \pi^{i}+C_{1 i} \pi^{i}+2 i C^{A} \bar{\eta}_{A}-2 i \bar{C}^{A} \eta_{A}-\left(\mathcal{D}_{i} C_{11}\right) \mu^{1 i}-C_{11} \mu^{1} \\
& +C_{1} M^{A} \bar{\eta}_{A}+C_{1} \bar{M}^{A} \eta_{A}+\frac{i}{2} C_{11} M^{A} \bar{\mu}_{A} \\
& \left.-\frac{i}{2} C_{11} \bar{M}^{A} \mu_{A}-C^{A} \bar{\mu}_{A} C_{1}-\bar{C}^{A} \mu_{A} C_{1}\right)
\end{aligned}
$$

comparing with (3.5) we see some other terms coming from the new choice of constraints. The BRST charge is again nilpotent when acting on the configuration space of the fields after the elimination of the auxiliary ones. The nilpotent BRST transformation laws are now

$$
\begin{aligned}
\widehat{\delta} M^{A} & =-2 i C^{A}-C_{1} M^{A}, \\
\widehat{\delta} C^{A} & =\frac{i}{2} C_{11} M^{A}-C^{A} C_{1},
\end{aligned}
$$

there are analogous changes for $\bar{M}^{A}$ and $\bar{C}^{A}$, while the transformation law for the other fields are as in (3.11). We define as before the SUSY transformations of $M^{A}$ and $C^{A}$. We have

$$
\begin{aligned}
\delta M^{A} & :=\left.\widehat{\delta} M^{A}\right|_{C_{1}=0} \\
\delta C^{A} & :=\left.\widehat{\delta} C^{A}\right|_{C_{1}=0}
\end{aligned}
$$

We then obtain

$$
\begin{aligned}
\delta \delta M^{A} & =-2 i \delta C^{A}=C_{11} M^{A} \\
\delta \delta C^{A} & =\frac{i}{2} C_{11} \delta M^{A}=C_{11} C^{A}
\end{aligned}
$$

as required. As shown the full SUSY algebra results from our nilpotent BRST charge. The BRST formulation using the combination of constraints (4.6) we have considered, leads to an effective action which may be obtained from (3.12) by a canonical change of coordinates in the original extended symplectic geometry. It is then a completely equivalent formulation.

\section{Conclusions}

In summary, we introduced a topological action with a large class of local symmetries, whose field equations are the Witten monopole equations found in [4]. By following a covariant gauge fixing procedure we obtained a covariant BRST invariant effective action . The BRST generator obtained is nilpotent off-shell. The canonical construction of the nilpotent BRST charge has been carried out without any further requirements on the base manifold beyond those assumed for the set up of action (2.1). 
This construction uses particular properties of the BRST charge for this topological theory, allowing a general canonical analysis on any compact 4-manifold $X$ without the standard assumption that $X=R \times \Sigma_{3}$. Finally we show how the twisted $\mathrm{N}=2$ supersymmetric algebra used to get the Witten topological theory may be directly obtained from our nilpotent BRST charge.

One of the main consequences of the existence of action (2.1) would be the possibility of relating the $S U(2)$ topological quantum field theory [1] directly to Witten's 4-monopoles theory [4]. In fact, the action (2.1) could be obtained by a partial gauge fixing that breaks the $S U(2)$ invariance to a $U(1)$ in the action already obtained in [3] for the $S U(2)$ topological theory together with some extra assumptions that we will discuss in a forthcoming communication. This would allow to compare directly the correlation functions of both topological theories by using the BFV theorem. This procedure seems interesting since does not use explicitly the duality relation between both theories found in [4] over a flat background nor it does use any supersymmetric argument. 


\section{APPENDIX}

Here we summarize the conventions used in this paper. To raise and lower spinor indices we use the matrix $C_{A B}$,

$$
C_{A B}=\left(\tau_{2}\right)_{A B}
$$

where $\tau_{i}, i=1,2,3$ are the Pauli matrices:

$$
\tau_{1}=\left(\begin{array}{ll}
0 & 1 \\
1 & 0
\end{array}\right), \quad \tau_{2}=\left(\begin{array}{cc}
0 & -i \\
i & 0
\end{array}\right), \quad \tau_{3}=\left(\begin{array}{cc}
1 & 0 \\
0 & -1
\end{array}\right)
$$

The inverse of $C_{A B}$ is given by $C^{A B}$ so that

$$
C^{A B} C_{D B}=\delta_{D}^{A}
$$

Lowering and raising spinors $\psi$ follow the rules

$$
\begin{aligned}
& \psi_{A}=\psi^{B} C_{B A} \\
& \psi^{A}=C^{A B} \psi_{B}
\end{aligned}
$$

To have a real $S_{\text {eff }}$ and a hermitian BRST charge $\Omega$ we define

$$
\begin{aligned}
& \bar{M}^{A}=M_{A}^{*} \\
& \bar{M}_{A}=-M^{A *} \\
& \eta^{A}=-\bar{\eta}_{A}^{*} \\
& \eta_{A}=\bar{\eta}^{A *}
\end{aligned}
$$

and for the even field $C^{A}$

$$
\begin{aligned}
& \bar{C}_{A}=C^{A *} \\
& \bar{C}^{A}=-C_{A}^{*}
\end{aligned}
$$

For even and odd objects with spinorial indices, we have the rule:

$$
\psi_{A} \bar{\phi}^{A}=-\psi^{A} \bar{\phi}_{A}
$$

We also use the set of four matrices $\sigma_{\mu}, \mu=0,1,2,3$, as

$$
\sigma_{0}=\left(\begin{array}{ll}
1 & 0 \\
0 & 1
\end{array}\right), \sigma_{a}=-i \tau_{a}^{T}, a=1,2,3
$$

and the matrices

$$
\widetilde{\sigma}_{\mu}^{A \dot{A}}=C^{A B} C^{\dot{A} \dot{B}} \sigma_{\mu B \dot{B}}=-C^{A B} \sigma_{\mu B \dot{B}} C^{\dot{B} \dot{A}}
$$




$$
\widetilde{\sigma}_{0}=-\left(\begin{array}{ll}
1 & 0 \\
0 & 1
\end{array}\right), \widetilde{\sigma_{a}}=-i \tau_{a}, a=1,2,3 .
$$

then we define $\sigma_{A}^{\mu \nu} \underset{A}{B}$ by:

$$
2 \sigma_{A}^{\mu \nu}{ }_{A}^{B}=g^{\mu \nu} \delta_{A}^{B}+\sigma_{A \dot{A}}^{\mu} \tilde{\sigma}^{\nu B \dot{A}}
$$

they satisfy the following properties:

$$
\begin{aligned}
\text { i) } & \sigma_{A}^{\mu \nu}{ }_{A}^{B} \sigma_{B \dot{B}}^{\lambda}=\frac{1}{2} g^{\lambda[\mu} \sigma_{A \dot{B}}^{\nu]}+\frac{1}{2} \epsilon^{\mu \nu \lambda \rho} \sigma_{\rho A \dot{B}} \\
i i) & \sigma_{A}^{\mu \nu}{ }_{A}^{B} \sigma_{B}^{\lambda \rho}=-\frac{1}{2} g^{\lambda[\mu} g^{\rho \nu]}-\frac{1}{2} \epsilon^{\mu \nu \lambda \rho} \\
i i i) & \sigma_{A B}^{\mu \nu} \sigma_{\mu \nu}{ }^{C D}=\delta_{(A}^{C} \delta_{B)}^{D}
\end{aligned}
$$

where $\epsilon^{0123}=-1$ and $A^{[\mu} B^{\nu]}=A^{\mu} B^{\nu}-A^{\nu} B^{\mu}$. The 2-form $B$ may be decomposed into its self-dual and anti-self-dual parts:

$$
B_{\mu \nu}=B_{\mu \nu}^{+}+B_{\mu \nu}^{-}
$$

where

$$
B_{\mu \nu}^{ \pm}=\frac{1}{2}\left(B_{\mu \nu} \pm \frac{1}{2} \epsilon_{\mu \nu \rho \sigma} B^{\rho \sigma}\right)
$$

we then have

$$
\begin{aligned}
& B_{A B}=a \sigma_{\rho \lambda A B} B^{\rho \lambda} \\
& B_{\mu \nu}^{+}=b \sigma_{\mu \nu}^{A B} B_{A B}
\end{aligned}
$$

where $2 a b=1$, so we may choose $a=\frac{1}{2}$ and $b=1$.

The full covariant derivatives in terms of the $U(1)$ connection and the covariant derivatives on the manifold $X$ are given by:

$$
\mathcal{D}_{A \dot{A}}=\sigma_{A \dot{A}}^{\mu} \mathcal{D}_{\mu}
$$

where

$$
\mathcal{D}_{\mu}^{ \pm}=\nabla_{\mu} \pm i A_{\mu}
$$

and $\nabla_{\mu}$ is the covariant derivative on the manifold $X$. 


\section{REFERENCES}

[1] E. Witten, Commun. Math. Phys. 117 (1988) 353.

[2] J.M.F. Labastida and M. Pernici, Phys. Lett. B212 (1988) 56.

L. Baulieu and I. M. Singer, Nucl. Phys. (Proc. Suppl.) 5B (1988) 12.

Y. Igarashi, H. Imai, S. Kitakado and H. So, Phys. Lett. B227 (1989) 239.

C. Aragão and L. Baulieu, Phys. Lett. B275 (1992) 315.

[3] R. Gianvittorio, A. Restuccia and J. Stephany, Phys. Lett. B347 (1995) 279.

[4] E. Witten, Math. Research Lett. 1 (1994) 769.

N. Seiberg and E. Witten, Nucl. Phys. B426 (1994) 19.

S. Hyun, J. Park, J-S. Park, hep-th/9508162.

[5] R. Gianvittorio,I. Martin and A. Restuccia, talk given at LASSF II, USB, Caracas October 1995

[6] M. Alvarez and J.M.F. Labastida, Nucl. Phys. B437 (1995) 356.

J.M.F. Labastida and Mariño, SLAC-PUB-US-FT 3/95.

J.M.F. Labastida and Mariño, hep-th/9507140.

[7] F. Hirzebruch and H. Hopf, Math. Ann. 136 (1958) 156.

[8] M.I.Caicedo and A. Restuccia, Class. Quan. Grav 10 (1993) 833.

M.I.Caicedo and A. Restuccia, Phys. Lett. B307 (1993) 77.

[9] M.Sohnius, Phys. Rep.128 (1985) 39-204. 